\title{
Evelyn Witkin and Stephen Elledge share the 2015 Lasker Basic Medical Award
}

$\mathrm{H}$ ic material? This year's Albert Lasker Basic Medical Research Award honors two distinct leaders in the field, Evelyn Witkin and Stephen Elledge, for their groundbreaking work to answer this question and uncover the cellular responses to DNA damage (Figure 1). Early in her career, Witkin isolated strains of UV-resistant bacteria and subsequently played a pivotal role in developing the SOS model of bacterial DNA damage response. A generation later, Elledge identified a number of key components of the signal transduction cascades that are critical for eukaryotic cells to sense and respond to damaged DNA and blocked DNA replication forks. Collectively, their work sheds light on multiple pathways that enable cells across the evolutionary spectrum to maintain genomic integrity.

\section{A pioneer in bacterial mutagenesis}

When Evelyn Witkin began her graduate studies in genetics at Columbia University in the early 1940s, DNA had not yet been definitively identified as the hereditary material $(1,2)$. Naturally curious, she was drawn to trying to understand central problems of genetics, which were how genes duplicate and how genetic changes are propagated. Inspired by a paper from Luria and Delbrück establishing that bacteria have genes and can develop viral resistance, Witkin pursued an opportunity to study bacterial genetics at Cold Spring Harbor Laboratory in the summer of 1944 (3). Her project was to mutate E. coli using ultraviolet light, but there were no published survival curves, and the initial doses she chose were quite high. "The next day, most of the plates were absolutely bare, no bacteria survived ... But on one plate there were four colonies," Witkin recalled (4). Suspecting that these isolates may have acquired UV resistance, she compared them with the parent strain and discovered that they had a higher tolerance to escalat-

ing doses of UV. "I had my first eureka experience, because these four colonies had arrived to what was clearly radiationresistant mutants, at least a hundred times more resistant to ultraviolet light than the original strain." She noted that normal strains exposed to UV stopped dividing and formed long filamentous structures, while resistant strains appeared to divide normally (5). She concluded at the time that the enhanced resistance observed was the result of spontaneous mutation.

Following the completion of her doctoral studies, Witkin remained at Cold Spring Harbor Laboratory until 1955. During these

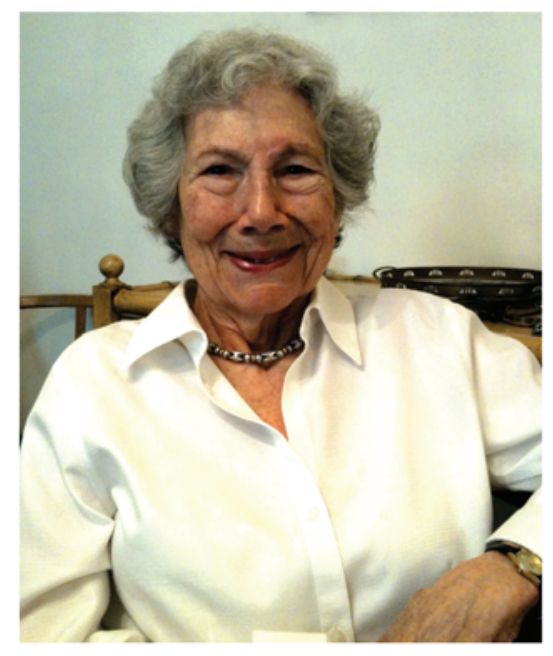

Figure 1. The recipients of the 2015 Albert Lasker Basic Medical Research Award. Evelyn Witkin (left), professor emerita at the Waksman Institute of Microbiology at Rutgers University and Stephen Elledge (right), Gregor Mendel Professor of Genetics in the Department of Genetics at Harvard Medical School and at Brigham and Women's Hospital.

years, she developed a close friendship with fellow geneticist Barbara McClintock. "I would have to say the most important experience I had there was being able to watch Barbara at work, to look over her shoulder during those years when she was discovering transposition. And this was with my mouth hanging open most of the time, because who could put this together with what we knew then about genetics?" (4).

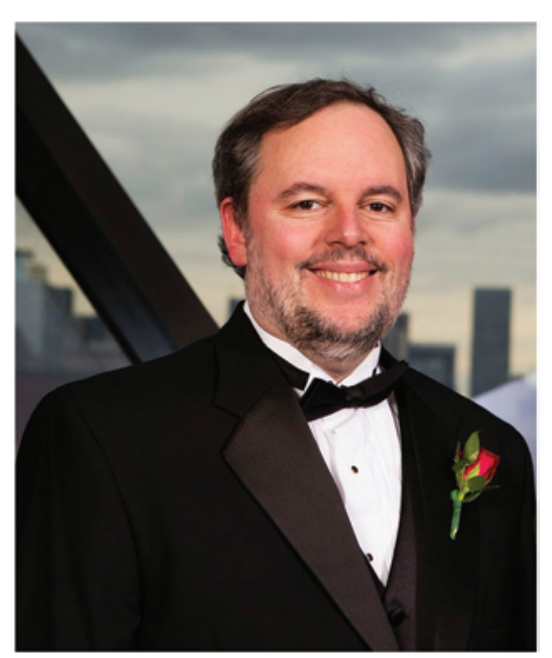

This close contact later influenced Witkin's own ideas, because she had learned from McClintock that broken chromosomes, even from disparate locations in the nucleus, can be rejoined efficiently, which helped shaped Witkin's thoughts about repair as a mechanism for resolving mutations.

\section{The SOS hypothesis}

After she left Cold Spring Harbor Laboratory, Witkin took a position at the State University of New York's Downstate Medical Center. With only a small laboratory space and a single technician, it was here that she began to develop a framework for the SOS 
A

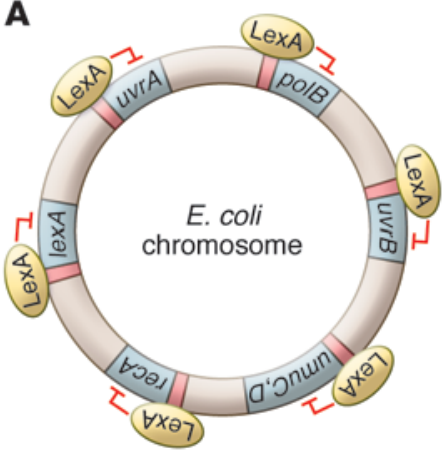

Figure 2. A simplified schematic of the bacterial SOS response. (A) Under normal conditions, LexA acts as a transcriptional repressor of over 40 downstream genes that mediate error-prone DNA replication and DNA repair, including $u v r A, u v r B$, and $u v r C$, which encode proteins that mediate nucleotide excision repair; $u m u C$, and $u m u D$, which encode subunits of the error-prone DNA polymerase $V$; and polB, which encodes another polymerase involved in the SOS response. (B) In the presence of DNAdamaging insults, such as UV light, the presence of single-stranded DNA triggers the formation of RecA filaments, which are important in the DNA strand-exchange reaction. RecA filaments also promote the autocatalytic cleavage of LexA, which derepresses genes involved in resolving DNA lesions. Evelyn Witkin's work provided a key framework for understanding the SOS response and predicted the presence of error-prone polymerases.

In addition, she returned to her early finding of a filamentous bacterial phenotype in response to UV and noted striking parallels with prophage induction (7). Prophage induction and filament formation are both induced by UV light or other DNA-damaging agents, and Witkin had observed that both occasionally occur spontaneously in old cultures. She proposed that $E$. coli contained a repressor, similar to the lambda phage repressor, that is inactivated by the presence of DNA damage that blocks replication fork progression and that can be restored following repair of the DNA lesion (7). In two additional reports, she found that bacterial strains with mutations in lexA, which we now know to encode a transcriptional repressor, or recA, which we now know to encode a single-stranded DNA-binding protein, could not produce UV-induced mutations (refs. 8, 9, and Figure 2). On the basis of these findings, Witkin suggested that these mutations might block the ability of an unknown DNA polymerase to catalyze error-prone replication.

In 1970, Miroslav Radman, a postdoctoral researcher at Harvard University, circulated a private memo coining the term "SOS replication" (10). In it, he hypothesized that lambda prophage induction and the activity of an error-prone DNA polymerase are under the control of the recA and lexA genes and suggested that DNA damage itself induces a distinct mecha- nism of DNA replication. Initially, Witkin had reservations about the model, but her own experiments would soon convince her that it must be correct. By now on the faculty of Rutgers University, she was working on a mutant $E$. coli strain lacking DNA polymerase I, which at the time was the only known DNA polymerase. She found that even at very low doses of UV exposure, this strain was hypermutable, and she recognized that this phenotype must be due to as predicted by the SOS hypothesis (11).

Shortly afterward, a number of prominent researchers linked by their investigations into recA- or lexA-dependent phenotypes gathered for a meeting in Gainesville, Florida (12). At this meeting, it became apparent that many of these fashion. It was here that Lorraine Gudas, a graduate student in Arthur Pardee's laboratory, proposed that LexA acted as a global repressor of the SOS response and that RecA promoted DNA damage-induced protease activity (13).

The race was now on to find genetic and biochemical evidence to support the SOS response hypothesis and to determine what other functions were induced by RecA. Subsequent work by Graham Walker's laboratory established a number of important loci that are expressed in response to DNA damage (14) and demonstrated that one of the downstream targets, the inducibility of an error-prone system, phenotypes were induced in a coordinated
UmuD, is also a target of RecA-mediated cleavage (15). The cleaved form of UmuD is now known to act as a subunit for a DNA polymerase $\mathrm{V}$, which plays a role in errorprone DNA replication. This research and many other studies that unfolded over the coming years thus supported the SOS model and eventually proved the existence of an inducible translesion polymerase, as predicted by Witkin.

\section{A new kid on the block}

It was during this exciting time of research that Stephen Elledge joined Graham Walker's laboratory as a graduate student in the Biology Department at MIT. His thesis studies focused on $u m u C$ and other bacterial genes that are inducible by DNA damage $(16,17)$. Though he had not met Witkin, his work genetically demonstrated that $u m u C$ is required for the error-prone repair process and added to the mounting evidence substantiating the SOS response.

During his postdoctoral studies in Ronald Davis' laboratory at Stanford University, Elledge originally intended to move away from the DNA damage response to instead focus on how to harness homologous recombination for reverse genetics in eukaryotic systems. $\mathrm{He}$ initially tried to find a protein with recombinase activity in Saccharomyces cerevisiae, but while searching for the yeast homolog of $r e c A$, a twist of fate sent Elledge down another path of research. The RecA antibody that he was using to identify a yeast homolog cross-reacted with a small subunit of ribonucleotide reductase (RNR2), which he discovered to be strongly inducible by DNA damage. The protein was approximately the right molecular weight, and he went on to clone the gene before he realized the error. "Of course, I was devastated ... It was just an artifact, and I wasn't at all interested in it," Elledge recalled to the JCI. This turned out to be a fortunate accident, though, because soon thereafter, Elledge learned that RNR2 is required during the cell cycle for DNA replication. From this, he deduced that cells must have a pathway that senses problems at the DNA replication fork and transmits this signal to promote $R N R 2$ transcription. This concept turned out to be correct, and Elledge showed that RNR2 expression is triggered by DNA damage and hydroxyurea, an agent that blocks DNA replica- 
UV damage or replication fork stalling

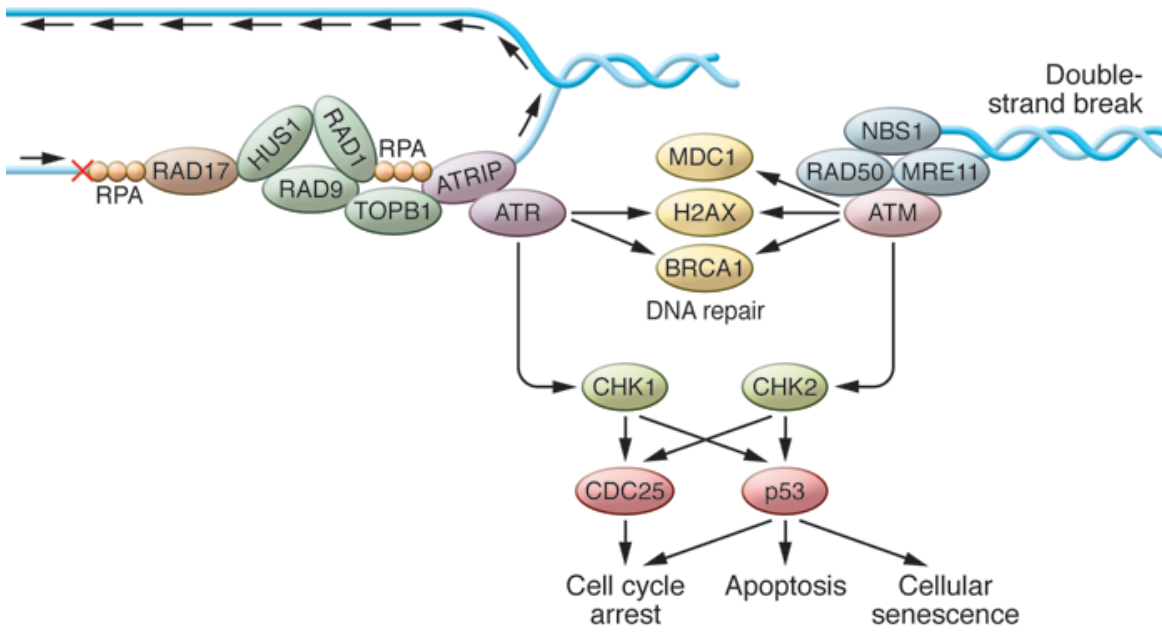

Figure 3. A simplified schematic of mammalian DNA damage response. In response to UV damage or replication fork stalling (left side), the presence of single-stranded DNA triggers binding by RPA, which independently recruits the ATR-ATRIP complex and the RAD17 complex. Subsequently, the 9-1-1 complex, composed of RAD9, RAD1, and HUS1, and TOPB1 are recruited, which in turn activates ATR. There are hundreds of downstream targets of the ATR kinase, including CHK1, BRCA1, and $\mathrm{H} 2 \mathrm{AX}$. This signaling cascade elicits a host of cellular responses, including DNA repair and cell cycle arrest. Improper resolution of DNA damage can trigger cell death or cellular senescence. Doublestrand breaks (right side) trigger the recruitment of the MRN complex, composed of MRE11, RAD50, and NBS1, which binds and activates ATM. Similar to ATR, ATM phosphorylates a host of downstream effectors to trigger DNA damage response pathways. Studies from Stephen Elledge's laboratory were instrumental in the understanding of how DNA damage is sensed and then leads to activation of downstream signaling cascades that trigger the DNA damage response.

tion (18-20). In addition, his work demonstrated that mutations in RNR2 conferred hypersensitivity to DNA replication stress, an area to which Elledge would devote much future research (19).

\section{An extraordinary period of discovery}

A year later, Leland Hartwell's laboratory published an article demonstrating that the $R A D 9$ gene is required for cell cycle arrest in $S$. cervisiae in response to x-ray irradiation (21). Similar studies in Schizosaccharomyces pombe had been published 12 years earlier in 1976 by Anwar Nasim's laboratory but were not widely known at the time (22). This rediscovery in budding yeast spurred considerable interest from the now burgeoning cell cycle field, because it further indicated that the cell negatively regulates the cell cycle in response to damage to permit cells more time to repair DNA. Elledge, by now running his own laboratory at Baylor College of Medicine in Houston, thought there might be a similar mechanism operating during the DNA synthesis phase of the cell cycle. He was convinced that there must be a signal transduction pathway that transmits information from replication stress or DNA damage to elicit both a cell cycle arrest and a broader transcriptional response of DNA damage-inducible genes. "But a lot of people in the field didn't believe this would be the case," Elledge noted. "They thought signal transduction came from the outside of the cell to the inside, and there really weren't any good examples of a cell sensing itself." To genetically test this, Elledge's first graduate student, Zheng Zhou, set up a reporter system to identify gene mutations that caused constitutive expression of RNR3 in the absence of DNA damage in $S$. cervisiae (23). They isolated a number of different mutants, including a pol1 mutant, which proved that blocking DNA replication altered the regulation of DNA damage-inducible genes.

Elledge and Zhou next set up the converse experiment to find gene mutations that blocked RNR3 expression following exposure to DNA-damaging agents. They identified several $d u n 1$ mutants that are sensitive to DNA damage and fail to induce expression of RNRs in response to damage (24). Importantly, the DUN1 gene encodes a kinase activated by DNA damage, and their study established for the first time that the eukaryotic DNA damage response is regulated by phosphorylation. They also noted that a subset of DNA damage-responsive genes were activated normally in the dun1 mutants, suggesting that other pathways must exist to control other aspects of the cellular response.

Elledge's laboratory would soon find evidence for an essential kinase that is critical for regulating the events downstream of replication stress. Jim Allen and others in the laboratory searched for yeast mutations that caused lethality in response to DNA replication blockade (25). They discovered that rad53 mutants exhibited blocked DNA damage-induced transcription of RNRs and failed to arrest at the G1, S, and G2 phases of the cell cycle in response to different damaging agents. $R A D 53$ encodes a protein kinase that acted upstream of DUN1, which confirmed the notion that signal transduction through a protein phosphorylation cascade is critical for mediating the ultimate cellular response to DNA damage.

\section{Extrapolating to mammalian cells}

While Elledge's laboratory was elegantly exploring the DNA damage response pathway in S. cervisiae, a number of other groups were trying to understand DNA damage deficiencies in mammalian cells. There was particular interest in a rare disorder known as ataxia-telangiectasia, which causes neurodegeneration, immune defects, and an increased susceptibility to cancer. In 1992, Michael Kastan and colleagues showed that primary cells from patients with ataxia-telangiectasia failed to induce the tumor suppressor p53 in response to ionizing radiation (26). Moreover, cells from patients with ataxiatelangiectasia also failed to transcriptionally upregulate GADD45, which had previously been implicated in the DNA damage response. This work provided an important line of evidence for a signal transduction network in response to DNA damage in human cells.

Soon afterward, Savitsky et al. cloned the human gene responsible for ataxiatelangiectasia (27). The ataxia-telangiectasia mutated gene $(A T M)$ was found to encode a kinase with similarity to PI3Ks, 
which were known to be involved in signal transduction. Just a few months later, Elledge's team identified two yeast homologs of ATM, MEC1 and TEL1, which they found controlled phosphorylation of RAD53 (28). They also predicted the existence of a human homolog of RAD53 that functions downstream of ATM.

In 1998, Elledge's laboratory proved that this assumption was correct, demonstrating that CHK2 is the mammalian equivalent of RAD53 and is phosphorylated by ATM in response to DNA damage (29). They also identified the human gene CHK1, which had homology to an S. pombe gene required for the DNA damage checkpoint (30). In collaboration with Helen Piwnica-Worms, they showed that CHK1 and, later, CHK2 could phosphorylate the CDC25 family of proteins, which inhibit cyclin-dependent kinases. They showed in S. cerevisiae that CHK1 and RAD53 operated in parallel pathways that are activated by different signals (31). Subsequently, the Elledge laboratory demonstrated genetically in mice that ATR functions upstream of CHK1 in a manner analogous to that of ATM and CHK2 (32).

These discoveries provided the backbone for much of our current understanding of how the eukaryotic cellular responses to DNA damage are mediated (Figure 3). In the ensuing years, Elledge's group continued to make seminal contributions to how DNA damage is sensed, discovering the association of ATR and ATR-interacting protein (ATRIP) and showing that this complex recognizes single-stranded DNA bound by RPA $(33,34)$. Their work also uncovered key mediators of the DNA damage checkpoint, including the breast cancer-associated protein BRCA1 and MDC1, which organizes DNA repair foci at sites of DNA damage $(35,36)$. With Tak Mak's laboratory, they also showed that induction of p53 in response to DNA damage is controlled by CHK2 (37). While many in the field contributed to our understanding of eukaryotic DNA damage response, Elledge's work stands out for mapping out the concept of how cells respond and systematically identifying many of the most important components.

One issue that had always vexed Elledge was that much of the field focused only on the cell cycle checkpoint aspect of the damage response. "I had to fight an uphill battle for 20 years to convince people that this was just one pathway and that the DNA damage response was controlling all kinds of different things," Elledge said. In 2007 he would provide compelling evidence to support this idea. A proteomic analysis of ATM and ATR substrates by Elledge's laboratory revealed that these two kinases phosphorylate hundreds of proteins, many of which had not been previously associated with the DNA damage response, including proteins involved in repair, metabolism, senescence, and apoptosis (38). This work, as Elledge predicted, clearly indicates that the DNA damage response network that cells utilize to cope with DNA damage is broad and extends far beyond its role in cell cycle checkpoints.

\section{A tribute to over 70 years of work}

From the simple observation of UV-resistant bacteria to our present understanding of the complex mechanisms underlying the DNA damage response, decades of work has uncovered several common features of this response across kingdoms, including sensing of DNA damage, the transmission of the damage signal through downstream mediators, the induction of cell cycle arrest, the transcription of factors required for repair, and the importance of feedback loops for detecting damage resolution. These pathways direct the ultimate cellular outcome, recovery in the case of successful repair, and cellular senescence or cell death in cases of irreparable damage. This year's Lasker Basic Medical Award pays tribute to two truly notable scientists, Evelyn Witkin and Stephen Elledge, who have played an exceptional role in shaping our understanding of these systems.

\section{Sarah Jackson}

1. Avery OT, MacCleod CM, McCarty M. Studies on the chemical nature of the substance induc ing transformation of pneumococcal types: induction of transformation by a desoxyribonucleic acid fraction isolated from Pneumococcus type III. J Exp Med. 1944;79(2):137-158.

2. Hershey AD, Chase M. Independent functions of viral protein and nucleic acid in growth of bacteriophage. J Gen Physiol. 1952;36(1):39-56.

3. Luria SE, Delbrück M. Mutations of bacteria from virus sensitivity to virus resistance. Genetics. 1943;28(6):491-511.

4. Conversations in Genetics presents talking with Evelyn Witkin [Video]. Youtube. https://www.
youtube.com/watch?v=ihJl12b6Lk4. Published March 15, 2015. Accessed August 21, 2015.

5. Witkin EM. Inherited differences in sensitivity to radiation in Escherichia coli. Proc Natl Acad Sci US A. 1946;32(3):59-68.

6. Witkin EM. Photoreversal and "dark repair" of mutations to prototrophy induced by ultraviolet light in photoreactivable strains of Escherichia coli. Mutat Res. 1964;106:22-36.

7. Witkin EM. The radiation sensitivity of Escherichia coli B: a hypothesis relating filament formation and prophage induction. Proc Natl Acad Sci US A. 1967;57(5):1275-1279.

8. Witkin EM. Mutation-proof and mutation-prone modes of survival in derivatives of Escherichia coli B differing in sensitivity to ultraviolet light. Brookhaven Symp Biol. 1967;20:17-55.

9. Witkin EM. The mutability toward ultraviolet light of recombination-deficient strains of Escherichia coli. Mutat Res. 1969;8(1):9-14.

10. Bridges BA. Error-prone DNA repair and translesion DNA synthesis. II: the inducible SOS hypothesis. DNA Repair (Amst). 2005;4(6):725-726.

11. Witkin EM, George DL. Ultraviolet mutagenesis in polA and UvrA polA derivatives of Escherichia coli B-R: evidence for an inducible error-prone repair system. Genetics. 1973;73(suppl 73):91-110.

12. Witkin EM. Ultraviolet mutagenesis and the SOS response in Escherichia coli: a personal perspective. Environ Mol Mutagen. 1989;14(suppl 16):30-34

13. Gudas LJ, Pardee AB. Model for regulation of Escherichia coli DNA repair functions. Proc Natl Acad Sci U S A. 1975;72(6):2330-2334.

14. Kenyon CJ, Walker GC. DNA-damaging agents stimulate gene expression at specific loci in Escherichia coli. Proc Natl Acad Sci U S A. 1980;77(5):2819-2823.

15. Nohmi T, Battista JR, Dodson LA, Walker GC. RecA-mediated cleavage activates UmuD for mutagenesis: Mechanistic relationship between transcriptional derepression and posttranslational activation. Proc Natl Acad Sci U S A. 1988; 85(6):1816-1820.

16. Elledge SJ, Walker GC. Proteins required for ultraviolet light and chemical mutagenesis. Identification of the products of the umuC locus of Escherichia coli. JMol Biol. 1983;164(2):175-192.

17. Elledge SJ, Walker GC. The muc genes of pKM101 are induced by DNA damage. JBacteriol. 1983;155(3):1306-1315

18. Elledge SJ, Davis RW. Identification and isolation of the gene encoding the small subunit of ribonucleotide reductase from Saccharomyces cerevisiae: DNA damage-inducible gene required for mitotic viability. Mol Cell Biol. 1987;7(8):2783-2793.

19. Elledge SJ, Davis RW. DNA damage induction of ribonucleotide reductase. $\mathrm{Mol} \mathrm{Cell} \mathrm{Biol.}$ 1989;9(11):4932-4940.

20. Elledge SJ, Davis RW. Identification of the DNA damage-responsive element of RNR2 and evidence that four distinct cellular factors bind it. Mol Cell Biol. 1989;9(12):5373-5386.

21. Weinert T, Hartwell L. The RAD9 gene controls the cell cycle response to DNA damage in Saccharomyces cerevisiae. Science. 
1988;241(4863):317-322.

22. Hannan MA, Miller DR, Nasim A. Changes in uv-inactivation kinetics and division delay in Schizosaccharomyces pombe strains during different growth phases. Radiat Res. 1976;68(3):469-479.

23. Zhou Z, Elledge SJ. Isolation of crt mutants constitutive for transcription of the DNA damage inducible gene RNR3 in Saccharomyces cerevisiae. Genetics. 1992;131(4):851-866.

24. Zhou Z, Elledge SJ. DUN1 encodes a protein kinase that controls the DNA damage response in yeast. Cell. 1993;75(6):1119-1127.

25. Allen JB, Zhou Z, Siede W, Friedberg EC, Elledge SJ. The SAD1/RAD53 protein kinase controls multiple checkpoints and DNA damageinduced transcription in yeast. Genes Dev. 1994;8(20):2401-2415.

26. Kastan MB, et al. A mammalian cell cycle checkpoint pathway utilizing p53 and GADD45 is defective in ataxia-telangiectasia. Cell. 1992;71(4):587-597.
27. Savitsky K, et al. A single ataxia telangiectasia gene with a product similar to PI-3 kinase. Science. 1995;268(5218):1749-1753.

28. Sanchez Y, Desany BA, Jones WJ, Liu Q, Wang B, Elledge SJ. Regulation of RAD53 by the ATM-like kinases MEC1 and TEL1 in yeast cell cycle checkpoint pathways. Science. 1996;271(5247):357-360.

29. Matsuoka S, Huang M, Elledge SJ. Linkage of ATM to cell cycle regulation by the Chk2 protein kinase. Science. 1998;282(5395):1893-1897.

30. Sanchez Y, et al. Conservation of the Chk1 checkpoint pathway in mammals: linkage of DNA damage to Cdk regulation through Cdc25. Science. 1997;277(5331):1497-1501.

31. Sanchez Y, et al. Control of the DNA damage checkpoint by chk1 and rad 53 protein kinases through distinct mechanisms. Science. 1999;286(5442):1166-1171.

32. Liu Q, et al. Chk1 is an essential kinase that is regulated by Atr and required for the G2/M DNA damage checkpoint. Genes Dev.
2000;14(12):1448-1459.

33. Cortez D, Guntuku S, Qin J, Elledge SJ. ATR and ATRIP: partners in checkpoint signaling. Science. 2001;294(5547):1713-1716.

34. Zou L, Elledge SJ. Sensing DNA damage through ATRIP recognition of RPA-ssDNA complexes. Science. 2003;300(5625):1542-1548.

35. Cortez D, Wang Y, Qin J, Elledge SJ. Requirement of ATM-dependent phosphorylation of brca1 in the DNA damage response to double-strand breaks. Science. 1999;286(5442):1162-1166.

36. Stewart GS, Wang B, Bignell CR, Taylor AMR Elledge SJ. MDC1 is a mediator of the mammalian DNA damage checkpoint. Nature. 2003;421(6926):961-966.

37. Hirao A, et al. DNA damage-induced activation of 53 by the checkpoint kinase Chk2. Science. 2000;287(5459):1824-1827.

38. Matsuoka S, et al. ATM and ATR substrate analysis reveals extensive protein networks responsive to DNA damage. Science. 2007;316(5828):1160-1166. 